\title{
Large-eddy simulation and low-order modeling of sediment-oxygen uptake in a transitional oscillatory flow
}

\author{
C. Scalo, ${ }^{1}$ L. Boegman, ${ }^{2}$ and U. Piomelli ${ }^{1}$ \\ Received 20 July 2012; revised 30 January 2013; accepted 5 February 2013.
}

[1] We have tested a dissolved oxygen (DO) transport model based on large-eddy simulation (LES) of a transitional oscillatory flow observed in the bottom boundary layer of Lake Alpnach, Switzerland. The transition from a quasi-laminar to a fully turbulent state makes this flow difficult to study with a Reynolds-averaged Navier-Stokes equation (RANSE) model. By resolving the full range of governing transport processes, LES provides a reliable prediction of the sediment-oxygen uptake (SOU). The model biogeochemical and flow parameters have been calibrated against DO and velocity measurements from published in situ data at the earliest phase available in the cycle. The fully developed flow thus obtained is used as an initial condition for the imposed oscillatory forcing. Numerical predictions show that transport in the outer layer is in equilibrium with the main current throughout most of the cycle and that nonequilibrium effects are limited to the diffusive sublayer response to the external forcing. During flow deceleration, the concentration boundary layer slowly expands as turbulence decays; later, during re-transition, mixing is restored by rapid and intense turbulent production events enhancing the SOU with a well-defined time lag. An algebraic model for the SOU is proposed for eventual inclusion in RANSE biogeochemical management-type models developed based on parameterizations used in turbulent mass transfer and with the support of published numerical data and the present simulation. The only input parameters required are the sediment oxidation rate, bulk temperature and DO concentration, and friction velocity.

Citation: Scalo, C., L. Boegman, and U. Piomelli (2013), Large-eddy simulation and low-order modeling of sediment-oxygen uptake in a transitional oscillatory flow, J. Geophys. Res. Oceans, 118, doi:10.1002/jgrc.20113.

\section{Introduction}

[2] The prediction of dissolved oxygen (DO) concentrations is critical for managing and monitoring marine ecosystems. Oxygen evolves in water bodies as a highSchmidt-number passive scalar with saturation levels dependent on temperature. It is entrained at the surface, produced by algae and plants, and transported vertically in the water column by turbulent mixing. In the sediment layer, decomposition of dead organic matter by oxygenconsuming bacteria can cause the DO concentration to drop to unsustainable levels for aquatic life. Therefore, the sediment-oxygen uptake (SOU) is the most important factor affecting oxygen depletion in the bottom mixed layer of water bodies [Patterson et al., 1985].

\footnotetext{
${ }^{1}$ Department of Mechanical and Materials Engineering, Queen's University, Kingston, Ontario, Canada.

${ }^{2}$ Department of Civil Engineering, Queen's University, Kingston, Ontario, Canada.

Corresponding author: C. Scalo, Center for Turbulence Research, NASA/Stanford University, Mechanical Engineering \& Institute for Computational Mathematical Engineering, Bldg. 500, Stanford University, Stanford, CA 94305-30035. (cscalo.ca@gmail.com, scalo@stanford.edu)

(C)2013. American Geophysical Union. All Rights Reserved. 2169-9275/13/10.1002/jgrc. 20113
}

[3] There are considerable environmental and economical impacts of oxygen depletion [Committee on Environment and Natural Resources, 2010]; however, the interactions among the processes involved in DO transfer across the sediment-water interface (SWI) and absorption by an organic sediment bed are not well understood. This knowledge gap hampers the fidelity of currently adopted predictive tools for ecosystem management [Koseff et al., 1993; Boegman et al., 2008a]. A systematic study of the physical processes leading to oxygen depletion is therefore required. These can be grouped into water-side and sediment-side transport [Grant and Marusic, 2011].

[4] Transport on the water side is governed by hydrodynamics. The velocity spectrum extends from basin-scale waves (order of kilometers) down to the Kolmogorov length scale, $\eta$ (order of centimeters or less). A high-Schmidtnumber scalar such as DO exhibits sub-Kolmogorov scales (smaller than $\eta$ ), which extend down to the Batchelor scale, $\eta_{B}$, typically of the order $\eta / 30$ for DO. Moreover, in the presence of a SWI, the molecular mass transfer is concentrated in a very thin diffusive sublayer (order of millimeters) [Wuest and Lorke, 2003] that is modulated by near-wall turbulent coherent structures [Scalo et al., 2012b]. The presence of roughness can also significantly alter the 
turbulent flow structure, changing the overall rates of mass and momentum transport [van Rijn, 1984].

[5] On the sediment side, a wide range of processes occur, ranging from biochemical reactions to hyporheic transport. The latter is defined as the exchange of solutes and momentum between water and sediments by means of advection [Grant et al., 2012]. Due to their inherent complexity and multi-scale nature, an empirical approach is often inevitable. The most important biochemical process governing oxygen depletion is bacterial absorption via organic matter decomposition, currently modeled as a nonlinear sink term proportional to the average bacterial population density $\chi$, which is not directly measurable [Higashino et al., 2008].

[6] In general, every solute dissolved in the pore water is transported by means of molecular diffusion, governed by porosity $\varphi$, mean advection and dispersion by pore water flow, governed by permeability $K$. Pore water flow is driven by nonuniform pressure distributions at the SWI [Riedl et al., 1972; Boudreau and Jørgensen, 2001; Higashino et al., 2009]. Depending on the sediment properties, the transport can be diffusion or advection dominated. In the presence of a sharp interface with cohesive sediments, diffusional transfer is expected to be dominant, while coarse-grain sediment beds may experience intense hyporheic exchange. Due to the interdependency of all of the aforementioned processes, a fully coupled approach is needed.

[7] Several complete (water and sediment) DO measurements are available at laboratory scale [e.g., O'Connor and Hondzo 2008]. Only a few numerical attempts, to our knowledge, have been made to reproduce the complete system with a fully coupled approach. These include Reynolds-averaged Navier-Stokes equations (RANSE) [Higashino et al., 2008; Higashino and Stefan, 2011] and large-eddy simulation (LES) [Scalo et al., 2012a] models. Both approaches have led to the development of models which have been calibrated to determine realistic estimates of the governing biogeochemical parameters (porosity, permeability, bacterial population); the LES has demonstrated high fidelity modeling of the unsteady small-scale processes, allowing the direct resolution of the near-wall turbulent coherent structures driving the hyporheic exchange and the mass transfer.

[8] The understanding acquired in the aforementioned lab-scale investigations can be used to interpret field-scale observations, for which extensive data are very onerous to collect. In Bryant et al. [2010], turbulence induced by an oscillatory current and the vertical distribution of DO above and within the sediments were measured and analyzed in Lake Alpnach, a lake in central Switzerland with pronounced seiching (i.e., presence of basin-scale standing waves with very long oscillation periods). The transient nature of the SOU is stressed, as well as its correlation with the phase-average near-wall turbulent intensity. This is the first attempt to correlate the mass transfer across the SWI to the large-scale dynamics and is a natural extension of the earlier work by Lorke et al. [2003] who focused on the near sediment region. Brand et al. [2008] have also investigated turbulent transport away from boundaries with the eddy-correlation (EC) technique, revealing a highly intermittent behavior of the vertical DO turbulent flux, sometimes exceeding (therefore, not in equilibrium with) the SOU. These observations are consistent with momentum and mass transport mechanisms present in turbulent oscillating boundary layers [Hino et al., 1983; Spalart and Baldwin, 1987; Costamagna et al., 2003].

[9] Despite the aforementioned experimental, numerical, and field work, there is currently no process-oriented model for the SOU that could take advantage of all the resolved quantities in RANSE models adopted for field-scale applications. The most advanced aquatic ecosystem model, to our knowledge, is the Computational Aquatic Ecosystem Dynamics Model (CAEDYM) [Hipsey and Hamilton, 2008]. Here a simple SOU model (referred to as static) is available where the DO flux is a function of the nearbed water temperature and DO concentration, bounded by an assigned maximum value. A more sophisticated SOU model (referred to as dynamic) is also available and incorporates a comprehensive characterization of the sediment geochemistry and organic matter diagenesis. Although the dynamic model includes Fickian-based solute flux (from the water side) at the SWI, there is no explicit dependency on near-wall flow parameters such as wall-shear stress or turbulent dissipation and, due to the numerous input parameters required, has never been fully validated. As the literature shows, the SOU depends heavily on the flow conditions [O'Connor and Hondzo, 2008; Scalo et al., 2012a]; if the flow is laminar or turbulent mixing is not fully established or is inhibited by other factors, the DO flux to the sediments can become negligible, given the very low molecular diffusivity of DO in water.

[10] In currently adopted models, the SOU is typically a case-dependent, tuneable parameter, fixed in time (and often in space) so that water-quality models can reproduce observations. The spatial and temporal variability of the (calibrated) DO flux at the SWI can, however, exceed an order of magnitude. In Trolle et al. [2008], the DO flux to the sediments is fixed to $0.7 \mathrm{~g} \mathrm{~m}^{-2} \mathrm{~d}^{-1}$ as a calibration parameter to match data for Lake Ravn, Denmark. In the work by Leon et al. [2006] in Lake Erie, a static model for the SOU is used with a maximum value of $0.6 \mathrm{~g} \mathrm{~m}^{-2} \mathrm{~d}^{-1}$ [Leon et al., 2012]. Boegman et al. [2008b] for Lake Erie used an SOU coefficient of $0.55 \mathrm{~g} \mathrm{~m}^{-2} \mathrm{~d}^{-1}$. Based on the formulation in Zhang et al. [2008], Conroy et al. [2011] for the same lake used a maximum SOU of $0.23 \mathrm{~g} \mathrm{~m}^{-2} \mathrm{~d}^{-1}$. Patterson et al. [1985] used $0.39 \mathrm{~g} \mathrm{~m}^{-2} \mathrm{~d}^{-1}$, obtained from Burns and Ross [1972], for central Lake Erie in August. Matisoff and Neeson [2005] report values in Lake Erie exhibiting more than an order of magnitude fluctuations (from 0.1 to $5.5 \mathrm{~g} \mathrm{~m}^{-2} \mathrm{~d}^{-1}$ ) due to seasonal and spatial variations. This variability is not captured in RANSE models where SOU is fixed in time and space (except for the temperature dependence in CAEDYM). The discrepancy between observed and model-adopted values, together with the complexity of field scale measurements, motivates the need to develop a simple time-space variable predictive model for SOU to be included in a RANSE code.

[11] In the present work, we adopt the eddy-resolving oxygen transport model developed by Scalo et al. [2012a] to reproduce numerically the processes leading to the SOU observed in the seiche-induced flow in Lake Alpnach studied by Bryant et al. [2010]. The goal is not to validate the model with the published field data but rather to design an idealized companion LES inspired by it. This allows 
us to generate self-consistent (and geophysically relevant) data to assist the development of a process-oriented lowerorder predictive model for the SOU, which is warranted in field-scale applications.

[12] The advantage of adopting an LES-based methodology lies in the ability to reproduce synthetically and analyze the instantaneous and three-dimensional structure of the velocity and concentration field, above the SWI and in the sediment layer. This provides reliable predictions under specified fluid dynamic and biogeochemical conditions and an accurate description of the physical processes involved. If the LES model were to be used to directly predict the field data analyzed here, initial and boundary conditions would have to be specified to the same (or higher) degree of accuracy and detail than provided by the LES model itself. While this proves to be impossible, comparison between the observations and LES still offers a very valuable contribution towards unraveling the fundamental physical processes involved in oxygen depletion driven by unsteady turbulence.

[13] In the following, we describe the complete transport model, based on LES, and the basic principles behind the proposed low-order model for the SOU. The computational setup adopted to model the hydrodynamic and biogeochemical conditions observed by Bryant et al. [2010] is also presented. Results from the LES are then shown together with the companion field data. Finally, the algebraic SOU model is empirically closed with data extracted from the present and previously published simulations and additional field data.

\section{Methodology}

\subsection{Large-Eddy Simulation of Oxygen Transfer to the Sediment Layer}

[14] LES is a well-established approach in the field of turbulent simulations allowing the prediction of turbulence characteristics for a wide variety of flows. The main idea is to adopt grids fine enough to resolve directly the evolution of the large turbulent scales that are strongly dependent on the initial and boundary conditions while modeling the residual mixing and dissipation occurring in the unresolved subgrid scales, which are supposed to exhibit universal characteristics [Richardson, 1922; Kolmogorov, 1941]. A direct numerical simulation (DNS) of a high-Schmidtnumber transported scalar such as DO would require a grid approximately 30 times finer in each direction than the Kolmogorov scale. This makes DNS prohibitive, especially for field-scale applications. LES is a feasible alternative since the scalar field and the velocity field share the same integral scale, and the effect of all subgrid scales (subKolmogorov and not) are accounted for in the model.

[15] The governing equations on the water side for the velocity field are the filtered continuity and Navier-Stokes equations [Leonard, 1974]:

$$
\begin{gathered}
\frac{\partial \bar{u}_{i}}{\partial x_{i}}=0 \\
\frac{\partial \bar{u}_{j}}{\partial t}+\frac{\partial \bar{u}_{i} \bar{u}_{j}}{\partial x_{i}}=-\frac{1}{\rho} \frac{\partial \bar{p}}{\partial x_{j}}-\frac{\partial \tau_{i j}}{\partial x_{i}}-f_{1} \delta_{1 j}-f_{3} \delta_{3 j}+v \nabla^{2} \bar{u}_{j}
\end{gathered}
$$

where $x_{1}, x_{2}$, and $x_{3}$ (or $x, y$, and $z$ ) are, respectively, the west-to-east, wall-normal, and north-to-south directions; $\bar{u}_{i}$ is the filtered velocity component in the $i$ th direction, and $\rho$ and $v$ are the fluid density and kinematic viscosity; and $\delta_{i j}$ is the Kronecker delta. The forcing terms $f_{1}$ and $f_{3}$ are the two mean pressure gradient components adjusted to achieve the desired flow rate in the $x$ and $z$ directions to reproduce the seiche dynamics.

[16] A top-hat filter is implicitly applied composed of three one-dimensional three-point discrete filters. The subgrid scale (SGS) stresses $\tau_{i j}=\overline{u_{i} u_{j}}-\bar{u}_{i} \bar{u}_{j}$ are parameterized by an eddy-viscosity model:

$$
\tau_{i j}-\delta_{i j} \tau_{k k} / 3=-2 v_{T} \bar{S}_{i j} \quad v_{T}=C \bar{\Delta}^{2}|\bar{S}|
$$

where

$$
\bar{S}_{i j}=\frac{1}{2}\left(\frac{\partial \bar{u}_{i}}{\partial x_{j}}+\frac{\partial \bar{u}_{j}}{\partial x_{i}}\right)
$$

is the filtered strain-rate tensor and $|\bar{S}|=\left(2 \bar{S}_{i j} \bar{S}_{i j}\right)^{1 / 2}$ is its magnitude; $\bar{\Delta}=\left(\Delta_{x} \Delta_{y} \Delta_{z}\right)^{1 / 3}$ is the filter width. The coefficient $C$ is evaluated using the dynamic procedure [Germano et al., 1991; Lilly, 1992] and averaged over directions of statistical homogeneity (in this case, planes parallel to the SWI).

[17] The oxygen concentration field is modeled as a highSchmidt-number passive scalar where the Schmidt number, $S c=v / D$, is the ratio between the kinematic viscosity of water and the molecular diffusivity of DO. The same SGS closure for the velocity field is used for the modeling of the unresolved turbulent scales of the DO field. The corresponding filtered conservation equation is

$$
\frac{\partial \bar{c}}{\partial t}+\frac{\partial \bar{u}_{i} \bar{c}}{\partial x_{i}}=\frac{\partial}{\partial x_{i}}\left[D \frac{\partial \bar{c}}{\partial x_{i}}-J_{i}^{s g s}\right] .
$$

The scalar is absorbed across the SWI by an organic sediment layer. The instantaneous DO field is transported by diffusion, advection, and dispersion but also depleted exclusively by decomposing organic matter according to

$$
\frac{\partial c_{s}}{\partial t}+\frac{\partial u_{s i} c_{s}}{\partial x_{i}}=\frac{\partial}{\partial x_{i}}\left(\left[F(\varphi) D+D_{e}\right] \frac{\partial c_{s}}{\partial x_{i}}\right)-\dot{c}_{s}
$$

The effects of porosity and tortuosity on diffusion in the pore water are accounted for in $F(\varphi)$ (which can be approximated by $\varphi^{3}$ for high porosities, $\left.\varphi>0.7\right)$, whereas dispersion effects are modeled by an effective diffusivity, $D_{e}$, calculated based on the parametrization proposed by Higashino and Stefan [2011] and, later on, extended to instantaneous quantities by Scalo et al. [2012a], resulting in

$$
D_{e}=\varphi^{2} d_{s}\left(u_{s j} u_{s j}\right)^{1 / 2}
$$

where $d_{s}$ is the sediment grain size, proportional to the square root of permeability via the Kozeny-Carmen relationship [Bear, 1972], and $u_{s i}$ is the $i$ th component of the interstitial velocity vector given by Darcy's Law

$$
u_{s i}=-\frac{K}{\mu_{v}} \frac{\partial p_{s}}{\partial x_{i}}
$$

where $\mu_{v}$ is the dynamic viscosity of water and $p_{s}$ is the pore-water pressure that satisfies a Laplace equation in the sediment layer. The parametrization for the nonlinear sink term, $\dot{c}_{s}$, is

$$
\dot{c}_{s}=\mu \frac{c_{s}}{K_{O_{2}}+c_{s}}
$$


Table 1. Bio-geochemical Parameters for Oxygen Absorption Model and Transport, in the Sediment Layer ${ }^{\mathrm{a}}$

\begin{tabular}{|c|c|c|c|c|c|c|}
\hline$\mu$ & $\mu_{0}$ & $\chi$ & $Y_{c}$ & $K_{O_{2}}$ & $\varphi$ & $K$ \\
\hline $240 \mathrm{mg} \mathrm{L}^{-1} \mathrm{~d}^{-1}$ & $2.4 \mathrm{~d}^{-1}$ & $100 \mathrm{mg} \mathrm{L}^{-1}$ & $1 \mathrm{mg}_{\chi} \mathrm{mg}_{\mathrm{DO}}^{-1}$ & $0.6112 \mathrm{mg} \mathrm{L}^{-1}$ & 0.94 & $2 \times 10^{-7} \mathrm{~cm}^{2}$ \\
\hline
\end{tabular}

where $\mu$ is the maximum oxidation rate that can be expressed as [Higashino et al., 2008; Higashino and Stefan, 2011],

$$
\mu=\frac{\mu_{0}}{Y_{c}} \chi
$$

where $\mu_{0}$ is maximum specific DO utilization rate (in $\mathrm{d}^{-1}$ ), $K_{\mathrm{O}_{2}}$ is the half-saturation coefficient for DO utilization (in $\mathrm{mg} \mathrm{L}^{-1}$ ), $Y_{c}$ is the effective yield for the microbial utilization of DO, and $\chi$ (in $\mathrm{mg} \mathrm{L}^{-1}$ ) is the value of biomass concentration of oxygen absorbing organisms, currently not directly measurable (Table 1 ). We assume a constant and uniform value of $\chi$ in the sediment layer. While the overall SOU is approximately proportional to this quantity, the oxygen concentration at the SWI exhibits a reduced sensitivity to $\chi$, varying less than $10 \%$ for changes of $100 \%$ in the bacterial population [Scalo et al., 2012a].

[18] The model has been validated by reproducing fluid dynamic conditions for different flow rates and temperatures in a laboratory flume where complete (water and sediment) DO measurements were obtained from microsensor data by O'Connor and Hondzo [2008]. The model relies on a new numerical strategy for the coupling of the discretized governing equations across the SWI, which allowed its extension to LES. Details regarding its implementation and validation can be found in Scalo et al. [2012a].

\subsection{Computational Setup}

[19] The computational setup used for the LES calculation is a turbulent open channel driven by two components of the pressure gradient (along $x$ and $z$ ) with an underlying DO absorbing organic sediment bed (Figure 1). The boundary conditions are periodic in the west-to-east $(x)$ and south-to-north $(-z)$ directions for all quantities. Homogeneous Neumann conditions are applied to the DO field at the bottom of the sediment layer $\left(y=-\delta_{s}\right)$ and at the top boundary $\left(y=L_{y}\right)$ on the water side; the instantaneous DO flux at the SWI is calculated by solving, at every time step, equation (6) in the sediment layer with the coupling strategy developed by Scalo et al. [2012a]. Free-slip conditions are applied to the velocity field at the top boundary, whereas velocites assigned at the SWI match the ones calculated by the pore-water-flow solver (equation (8)) in the sediment layer (which are negligible, for this case, with respect to the friction velocity). The flow is initialized at time $t=0$ with an instantaneous velocity and scalar field obtained from a statistically steady simulation, and the seiche forcing is reproduced by dynamically adjusting the mean pressure gradient to match the measured current intensity in the south-to-north and west-to-east directions. After time $t=0$, the initial concentration field is left to decay, with no topboundary re-aeration and with an instantaneous absorption rate modulated by the transient flow. The simulation covers

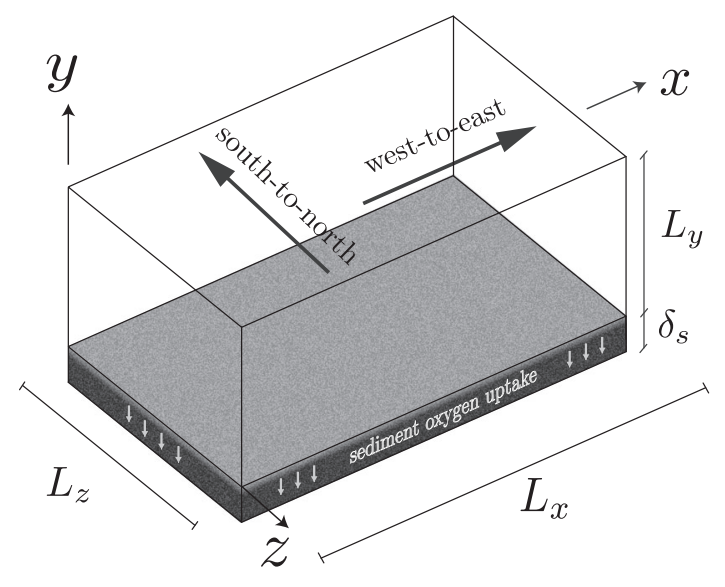

Figure 1. Sketch of a three-dimensional doubly-periodic open channel with an oxygen absorbing sediment layer. Domain size is $L_{x}=300 \mathrm{~cm}, L_{y}=52 \mathrm{~cm}, L_{z}=150 \mathrm{~cm}$, and sediment layer thickness $\delta_{s}=1 \mathrm{~cm}$.

$11 \mathrm{~h}$ of physical time, during which the bulk oxygen concentration decays by $3 \%$. See sections 3.1 and 3.2 .1 for a detailed discussion on the derivation of the initial conditions for the LES and the simulation of the seiche-induced hydrodynamic forcing.

[20] The vertical extent of the resolved water column $L_{y}$ has been chosen so that $R e_{\tau, 0}=u_{\tau, 0} L_{y} / v=400$ (where $u_{\tau, 0}$ is the total friction velocity at $t=0$ ). This results in $L_{y}=52 \mathrm{~cm}$. Simulating a shorter water column would have the effect of excessively reducing the size of the outer layer scales, introducing low Reynolds numbers effects. A taller domain would increase the size of largest resolved eddies (in the outer layer), increasing the overall computational cost (more grid points would be needed in $y$ ). This would not significantly benefit the present calculation, given the mild influence of the outer layer on the near-wall turbulence, which for $R e_{\tau, 0}>400$ starts to exhibit Reynolds number invariance [Moser et al., 1999]. The near-wall structures, if normalized by $u_{\tau, 0}$ and $v$ (given quantities in our problem), exhibit universal characteristics that are (approximately) unchanged by the Reynolds number (or, in this case, by the choice of $L_{y}$ ). Moreover, the near-wall transport events are the only ones that need to be reproduced accurately to predict the DO flux across the SWI [Scalo et al., $2012 \mathrm{~b}]$. The extension of the computational domain in the other two directions, $L_{x}$ and $L_{z}$, is chosen to accommodate the largest scales and the near-wall structures.

[21] The numerical model used to compute the flow on the water side is a well-validated finite-difference code [Keating et al., 2004], based on a three-dimensional Cartesian staggered grid. Second-order central differences 
are used for both convective and diffusive terms. The time advancement scheme is Crank-Nicholson for the wallnormal diffusive term, and low-storage three-step secondorder Runge-Kutta for the other terms. The solution of the Poisson equation is obtained by Fourier transform of the equation in the spanwise and streamwise directions, followed by a direct solution of the resulting tridiagonal matrix, at each wave number. The code is parallelized using the MPI protocol. The equations in the sediment layer are solved with the same numerical approach as the one adopted for water side and have the same accuracy and stability properties.

[22] The grid resolution at $t=0$ is $\Delta x^{+}=18$ and $\Delta z^{+}=$ 11 where ()$^{+}$indicates lengths normalized by $v / u_{\tau}$. A high resolution is required to account for the large excursion in the wall-shear stress (resulting in a highly variable turbulent state, and the presence of turbulence decay and re-transition) and to resolve accurately the high-Schmidt-number passive scalar field. This results in a grid size of approximately $\Delta x=2.3 \mathrm{~cm}$ and $\Delta z=0.8 \mathrm{~cm}$. The minumum grid spacing in the wall-normal direction is $\Delta y=0.01 \mathrm{~cm}$ at the SWI (to resolve the diffusive sublayer). The Navier-Stokes solver time step varied in the range of $0.01-0.1 \mathrm{~s}$.

\subsection{An Algebraic Parametrization for the Sediment-Oxygen Uptake}

[23] In our LES-based numerical model, the DO flux to the sediment layer is an output of the computation. Conversely, in RANSE models used at field scale, the SOU is typically an input parameter (a boundary condition) and very little information on the state of the near-wall turbulence (or the bed properties) is available to model it accurately. This may include a Reynolds stress, dissipation or eddy viscosity computed as an average over a time step and grid cell of the order of minutes and kilometers, respectively. Spatial resolution is also an issue. The vertical extent of the first computational cell from the SWI used in field-scale numerical models is typically comparable to (if not larger than) the entire height of the computational domain used here $\left(L_{y}=52 \mathrm{~cm}\right)$. The resolution required to accurately resolve the high-Schmidt-number transport mechanisms in such a limited domain (Table 2) makes a wall-resolved LES an unfeasible option for field-scale applications. The development of a process-oriented algebraic model to estimate the SOU for eventual inclusion into a RANSE (or wall-modeled LES) geophysical code is therefore warranted. The fundamentals of the new proposed SOU model are discussed in the following.

[24] The model focuses on the prediction of the waterside diffusion-limited component of the oxygen flux across the interface relying only on the few near-wall volumeaveraged quantities that are available at run-time in geophysical codes and minimal biogeochemical information of the sediment layer (very difficult to measure). These include the bulk velocities in the plane parallel to the bed, $U_{B}$ and
$W_{B}$, temperature $T_{B}$ and oxygen concentration $C_{\infty}$, and the maximum oxidation rate $\mu$.

[25] Based on parameterizations available in the literature for high-Schmidt-number mass transfer [Boudreau and Jørgensen, 2001] , mostly valid under equilibrium conditions, the diffusive component of the DO flux across the SWI can be expressed as

$$
\mathrm{SOU}=\beta \Delta C
$$

where $\beta$ is a (dimensional) mass transfer coefficient and $\Delta C$ is the oxygen concentration defect (driving chemical potential for the flux) at the SWI

$$
\Delta C=C_{\infty}-C_{s w i}
$$

where $C_{s w i}$ is the mean oxygen concentration at the SWI. The normalization for the DO implied by equation (11) is consistent with the linearity of the transport equation for the scalar field on the water side. Both the terms on the right-hand side of equation (11) need to be modeled with parameters dynamically resolved in the simulation and with some sediment-specific information.

[26] Scalo et al. [2012b] have verified that for steady hydrodynamic conditions and flat cohesive sediment layers, the dependency of the SOU (equation (11)) on the sediment characteristics (in particular, the maximum oxidation rate $\mu$ ) can be removed if normalizing it with the concentration difference $\Delta C$. This result can be extended to a more general case where the DO field exhibits a two-layer structure about the SWI, which is typically observed in the case of oxygen transport. The quantity SOU/ $\Delta C$ is the mass transfer coefficient $\beta$, which is modeled solely based on the hydrodynamics. This suggests that $\Delta C$ is the only term in equation (11) that is directly affected by the bio-geochemical properties of the sediment layer.

[27] This can be interpreted from a mathematical perspective. Due to the passive nature of the transported scalar on the water side, the same turbulent flow with different underlying sediment layers, for example, one with low and one with high oxidations rates (low and high values of $\Delta C$, respectively), will exhibit identical instantaneous mixing dynamics determined uniquely by the velocity field. In the highly absorbing case, the same mixing events lead to a higher flux simply due to the presence of a stronger background concentration gradient (higher $\Delta C$ ). From a mathematical standpoint, changing the oxidation rate or the sediment effective diffusivity (for example, by changing the porosity and/or permeability) will only affect the boundary conditions (via $\Delta C$ ) of the corresponding Reynoldsaveraged scalar transport equations (Figure 2). Given their linearity and homogeneity in the scalar concentration field, their solutions collapse into one if scaled by $C_{s w i}$ and normalized by $\Delta C$. As long as the sediment characteristics do

Table 2. LES Parameters ${ }^{\mathrm{a}}$

\begin{tabular}{lccccccccc}
\hline$L_{x}(\mathrm{~cm})$ & $L_{z}(\mathrm{~cm})$ & $L_{y}(\mathrm{~cm})$ & $\delta_{s}(\mathrm{~cm})$ & $N_{x}$ & $N_{y}$ & $N_{z}$ & $N_{y, s}$ & $v\left(\mathrm{~cm}^{2} / \mathrm{s}\right)$ & $D\left(\mathrm{~cm}^{2} / \mathrm{s}\right)$ \\
\hline 300 & 150 & 52 & 1 & 128 & 256 & 192 & 48 & $1.39 \times 10^{-2}$ & $1.36 \times 10^{-5}$ \\
\hline
\end{tabular}

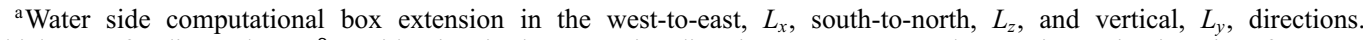
Thickness of sediment layer, $\delta_{s}$. Grid points in the respective directions $N_{x}, N_{z}, N_{y}$, and $N_{y, s}$. Kinematic viscosity of water $v$ and molecular diffusivity of oxygen, $D$. 


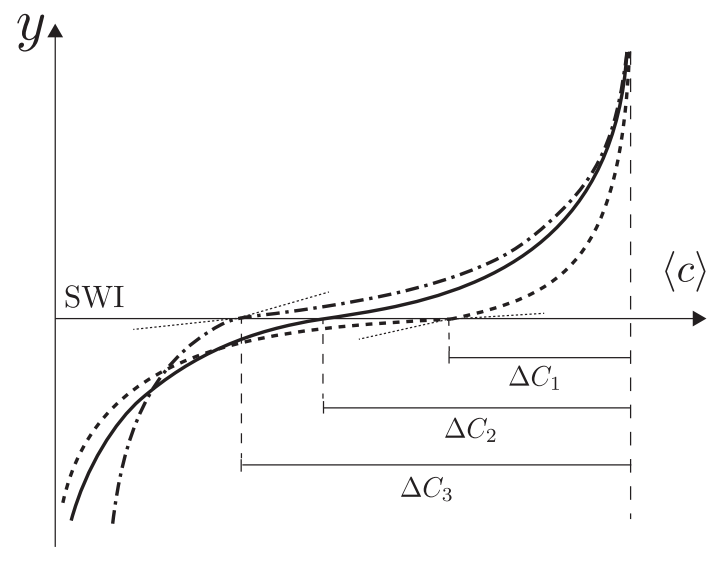

Figure 2. Effects of sediment porosity, $\varphi$, and dispersivity (controlled by permeability $K$ ) on oxygen profiles: (1) Purely diffusive sediment layer with low porosity (---), (2) porosity approaching 1 , no dispersive effects ( - , (3) dispersive-dominated transport (-. - ). The gradient discontinuities are highlighted with the finely dotted lines. The effects on $\Delta C$ are exaggerated for visualization purposes.

not affect significantly the structure of the overlying turbulent velocity field, the flux at the SWI is proportional to $\Delta C$ and $\beta$ is only dictated by the hydrodynamics (as commonly done in practice [Boudreau and Jørgensen, 2001]). In conclusion, equation (11) is consistent with the classic interpretation of $\beta$ as a measure of the efficiency of the nearwall turbulent mixing working against a given background concentration difference $\Delta C$.

[28] At this stage, closures for $\beta$ and $\Delta C$ need to be derived. The mass transfer coefficient can be expressed as a function of the Schmidt number, $S c$, and the total friction velocity, $u_{\tau}$, according to

$$
\beta=u_{\tau} A S c^{-n}
$$

where $A$ and $n$ are fitting coefficients retrievable from published works, for example, Shaw and Hanratty [1977]. The Schmidt number can be calculated from the near-wall average water temperature, $T_{B}[L i$ and Gregory, 1974], while the friction velocity can be calculated either from the bulk or free stream velocities, via the bottom-drag coefficient [Wuest and Lorke, 2003] or by using a log-law fit of the mean velocity profile. These strategies for predicting the wall-shear stress are tested in section 3.2.1 for the flow under investigation.

[29] Although $\beta$ depends solely on the hydrodynamics, the concentration difference $\Delta C$ is still dependent on all the governing parameters and is indicative of the oxygen content of the sediment layer. In equilibrium conditions, $\Delta C$ is determined by a balance between the rate of absorption in the sediment layer and the DO transfer rate from the water side. It is shown that high-Schmidt-number mass transfer mechanisms are intimately connected to viscous sublayer turbulent velocity fluctuations [Pinczewski and Sideman, 1974; Scalo et al., 2012b] and that the latter scale in viscous units (based on $v$ and $u_{\tau}$ ) fairly accurately [Moser et al., 1999]. These considerations lead to the following expression for the normalized concentration difference at the SWI:

$$
\text { where } \quad \begin{aligned}
\Delta C / C_{\infty} & =f\left(\mu^{+}, S c, \varphi, K^{+}\right) \\
\mu^{+} & =\frac{\mu \nu}{C_{\infty} u_{\tau}^{2}}
\end{aligned}
$$

is the ratio between the characteristic near-wall (viscous) time scale $t_{\nu}=v / u_{\tau}^{2}$ and the oxidation time scale $t_{\mu}=$ $C_{\infty} / \mu$, and $K^{+}$is the intrinsic permeability normalized with the viscous length scale, $\delta_{v}=v / u_{\tau}$. In the following, the SOU predictions extracted from the present simulation and previously published lab-scale LES are used to derive an explicit functional form for equation (14). The SOU modeled by equation (11) represents the actual DO flux across the SWI and not the potential sediment-oxygen demand (PSOD), which is exclusively a function of the biochemistry of the sediment layer (see Introduction of Bryant et al. [2010]), that would be attained with no limitation on the oxygen supply from the water side.

\section{Results and Discussion}

\subsection{Initial Conditions for LES}

[30] In LES of unsteady and transitional flows, like the one analyzed in this work, predictions are highly dependent on the turbulent field used as an initial condition. By starting the simulations from a previously developed fully turbulent and statistically steady flow, we ensure that the simulated flow, in the time range of interest (between $t=0$ and $t=11 \mathrm{~h}$ ), is always physical. The resulting fluid dynamic conditions at $t=0$ are, therefore, of equilibrium turbulence and might not exactly correspond to the actual flow conditions in the field at that time. Real-world flows include a wide range of complexities, knowing that the true initial conditions and boundary conditions to the accuracy required by eddy resolving methods such as LES is very difficult and, for the purpose of this work, not necessary.

[31] A statistically steady turbulent velocity field (Figure 3a) is, therefore, first obtained by running the numerical model until statistical convergence is reached. The fluid dynamic conditions of this field correspond to those observed at $10 \mathrm{pm}$ in Bryant et al. [2010]. We set this as $t=0$ in the present calculation. The mean velocity field was reconstructed by applying the log-law to the west-toeast and south-to-north velocity components measured at $y=10 \mathrm{~cm}$ (Figure 4a). The flow rates in the corresponding directions were estimated via analytical integration of these velocity profiles and were then imposed by adjusting the orientation and magnitude of the mean pressure gradient. The resulting total friction velocity at time $t=0$ is written as $u_{\tau, 0}=\left(\tau_{w, 0} / \rho\right)^{1 / 2}$, where $\tau_{w, 0}=\left(\tau_{w, 0 x}^{2}+\tau_{w, 0 z}^{2}\right)^{1 / 2}$ is the magnitude of the total wall-shear stress. At this time, $\tau_{w, 0 z}$ is negligible with respect to $\tau_{w, 0 x}$, but this is not the case for the rest of the seiche cycle (Figure $4 \mathrm{~b}$ ).

[32] The turbulent DO distribution used as an initial condition (Figure $3 b$ ) is obtained from the same statistically steady simulation used to initialize the velocity field; $\mu$ and $K$ have been adjusted to match the DO measurements at $10 \mathrm{pm}$; a Schmidt number of $S c=1024$, consistent with water temperatures of $T=8 \mathrm{C}^{\circ}$, and a porosity of $\varphi=0.94$ (as suggested in Bryant et al. [2010]) have been used. A free stream DO concentration of $C_{\infty}=3.84 \mathrm{mg} \mathrm{L}^{-1}$, taken 

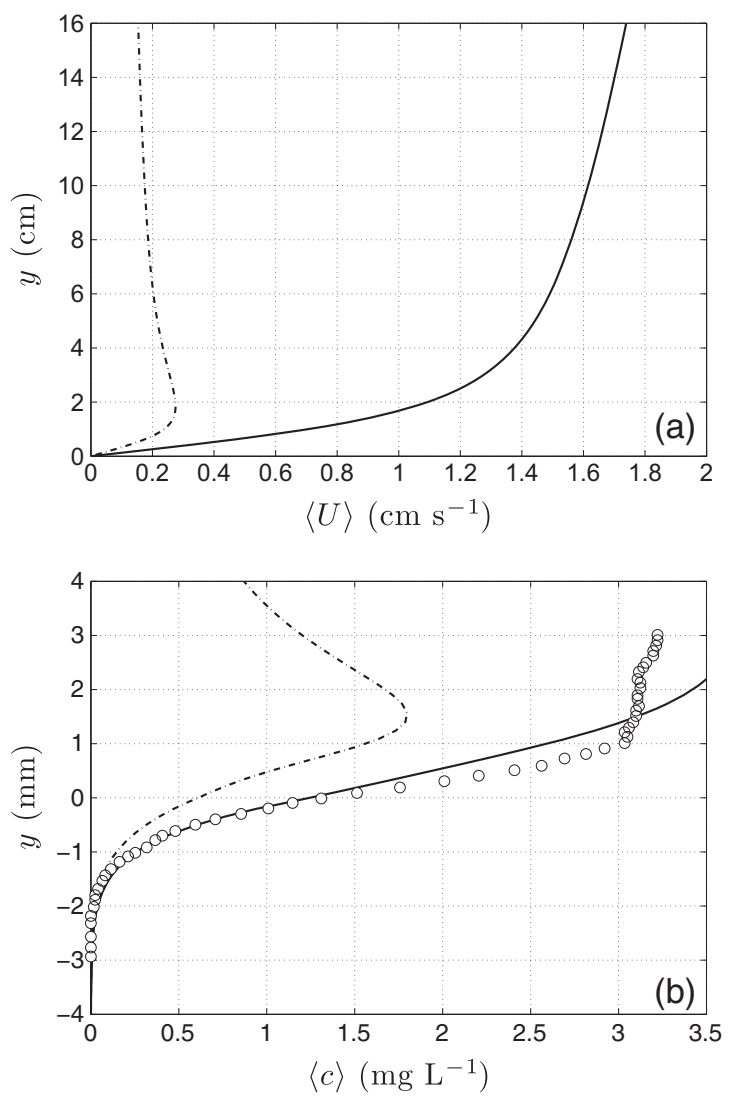

Figure 3. Profiles of mean (-) and resolved RMS (- - - ) of (a) west-to-east velocity component and of (b) DO at time $t=0$. Corresponding oxygen measurements obtained via microprofiling by Bryant et al. [2010] (o) at $10 \mathrm{pm}$.

from the maximum value observed in the microsensor data, is used at $t=0$. For these parameters, $\chi=100 \mathrm{mg} \mathrm{L}^{-1}$ returned the best match inside the sediment layer at the initial time $t=0$ under steady-state fluid dynamic conditions. The gradient discontinuity across the SWI in measured oxygen profiles is not visible at every phase of the cycle due to the high porosity (approximately 1; see Table 1). It is, however, mostly consistent with an overall diffusivity that is slightly higher in the pore water than on the water side (see Figure 5 of Bryant et al. [2010] and Figure 3b). This can only be explained assuming a dispersive behavior of the sediment layer [Scalo et al., 2012a] (Figure 2). Finally, a value of $K=2 \times 10^{-7} \mathrm{~cm}^{2}$ was determined from the calibration and, despite being very low, is retained in the calculation.

[33] For the flow conditions at $t=0$ (Figure 3), the peak in the streamwise RMS of velocity occurs $y=1.8 \mathrm{~cm}$ above the SWI, where buffer-layer turbulent transport events occur. These are responsible for governing the high-Schmidtnumber mass transfer across the SWI and sustaining the scalar variance profile (peaking at $y=1.5 \mathrm{~mm}$, approximately the edge of the diffusive sublayer). At this time, the gradient of the oxygen distribution appears to be continuous across the SWI; in reality, the enhanced diffusivity due to dispersion effects tends to increase the mixing in the pore water and reduce the overall DO gradient. This effect is partially compensated by the slightly reduced molecular

\section{(a)}

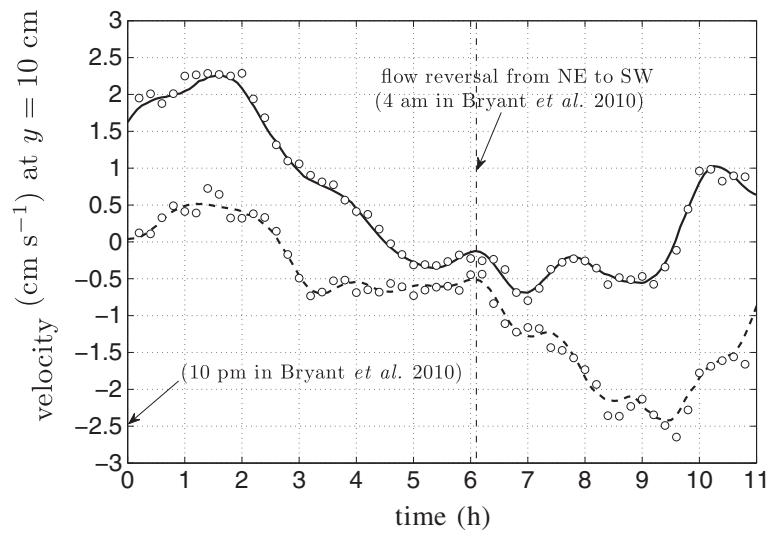

(b)

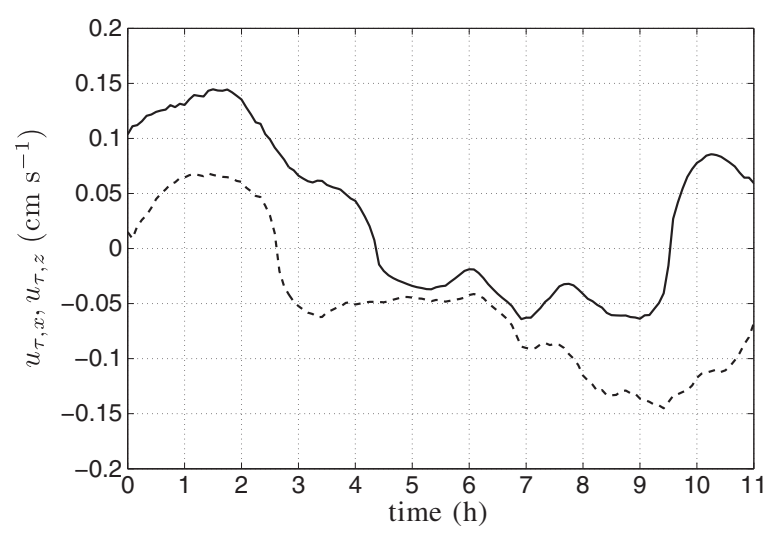

Figure 4. Time series of mean current velocity at $10 \mathrm{~cm}$ from (a) the SWI and (b) corresponding friction velocities, west-to-east $\langle u\rangle(-)$ and south-to-north $-\langle w\rangle$ (-- ) components predicted by LES and measurements by Bryant et al. [2010] (o) via acoustic Doppler velocimeter (ADV).

diffusion in sediment layer. The diffusive sublayer predicted by the calibrated LES is slightly thicker than the one observed in the measurements. A better agreement with the oxygen measurements on the water side could be obtained by decreasing $\chi$, increasing the Reynolds number or increasing $K$. In the first case, the oxygen levels in the sediment layer would be significantly overestimated, given the relatively reduced sensitivity of the oxygen distribution on the water side to variations in $\chi$ [Scalo et al., 2012a]. Increasing the Reynolds number would improve the agreement for $0<y<1 \mathrm{~mm}$ by reducing the diffusive sublayer thickness. This would, however, require the adoption of physical parameters (water temperature, viscosity, current velocity, etc.) not consistent with the flow conditions in Bryant et al. [2010] and, therefore, not justifiable. The same effect on the water side can be obtained by increasing the sediment layer permeability. This would increase the effective diffusivity and, therefore, increase the DO flux (Figure 2). While this would improve the agreement on the water side, large discrepancies would appear in the pore water DO distribution. Moreover, the measured mean oxygen distribution does not suggest the presence of significant dispersion. Its effect on the DO dynamics can, therefore, be regarded as marginal with respect to the seiche forcing. 
(a)

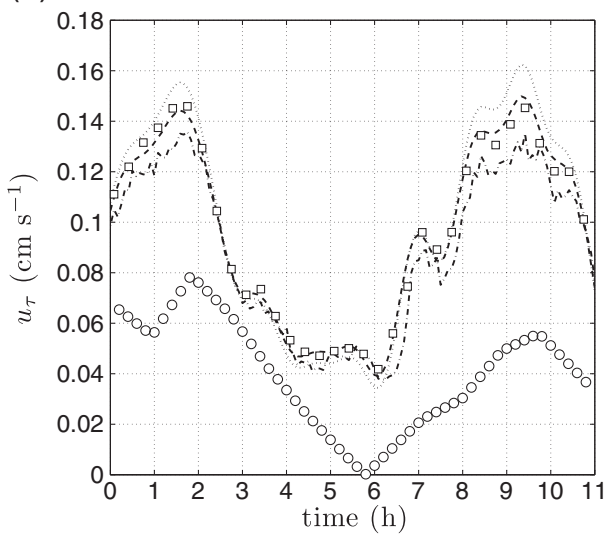

(b)

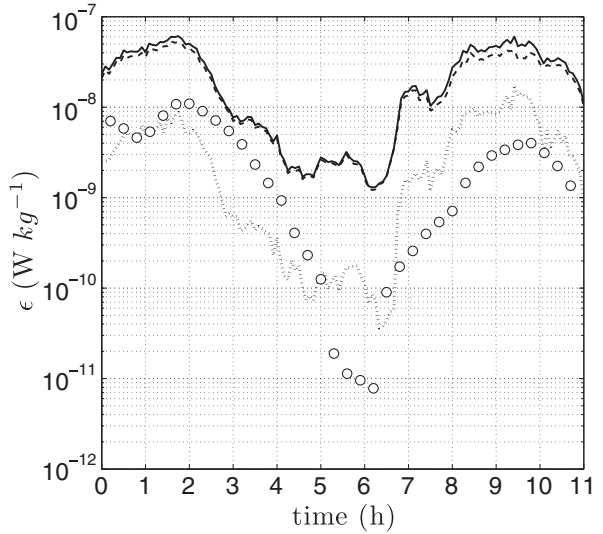

Figure 5. (a) Comparison of different methods to evaluate the total friction velocity $u_{\tau}$ : direct calculation from LES $(\square)$, log-law fit of mean velocity magnitude profile (---), bulk velocity bottom-drag coefficient of $C_{B}=0.0036$ (17) (…...), law-of-the-wall equation (16) applied to total dissipation calculated from the LES at $y=10 \mathrm{~cm}(-\cdot-)$, and estimates by Bryant et al. [2010] by applying law-of-the-wall to dissipation measurements (shown in the panel below) (o). (b) Turbulent kinetic energy viscous dissipation at $y=10 \mathrm{~cm}$ : total (—), resolved (---), and SGS (…...) dissipation from LES (the SGS component of the dissipation is not directly resolved but modeled via equation (3); the total dissipation is the sum of the resolved and SGS component), measurements by Bryant et al. [2010] (o) obtained by inertial dissipation method using longitudinal, planar, and vertical velocity data as measured via acoustic Doppler velocimeter (ADV).

[34] An interesting feature of the measured oxygen profiles, also observed in lab-scale measurements [O'Connor and Hondzo, 2008], is the gradient discontinuity visible, on the water side, at $y \simeq 1 \mathrm{~mm}$. This is present in many of the measured profiles (shown later). This discontinuity could be explained by an abrupt variation of the eddy diffusivity (from zero, for $y<1 \mathrm{~mm}$, to a finite value, for $y>1 \mathrm{~mm}$ ), which cannot be found in fully developed flows. One possibility is that the turbulent concentration boundary layer is at its early stages of spatial development, and the sharp variation in the mean gradient is actually determined by the upwardly propagating front of concentration defect. An accurate knowledge of the local spatial distribution of the bacterial population at the SWI and in the sediment layer, very difficult to achieve at field scale, would be required to improve the quality of the predictions.

\subsection{Seiche Dynamics}

\subsubsection{Wall-Shear Stress and Dissipation}

[35] The flow rates imposed at time $t=0$ along $x$ and $z$ (calculated assuming the validity of the log-law) are varied for $t>0$ proportionally to the measured velocities at $y=10$ $\mathrm{cm}$ by adjusting dynamically the pressure gradient terms $f_{1}$ and $f_{3}$ in equation (2). In spite of the approximations made, the predicted wall-shear stress components in the westto-east and south-to-north directions follow very closely the measured current velocities at $y=10 \mathrm{~cm}$ (Figure 4). This suggests that the equilibrium assumption (made on the velocity field when deriving the initial conditions for the LES) can be extended with good approximation to most of the cycle. This has also been confirmed by Bryant et al. [2010] except near flow reversal where current velocities at $y=10 \mathrm{~cm}$ fall below $1 \mathrm{~cm} \mathrm{~s}^{-1}$.
[36] The corresponding total friction velocity estimated by Bryant et al. [2010] (Figure 5a), however, does not follow the variations in the measured current velocity at $y=10 \mathrm{~cm}$ to the same extent the LES does. It was estimated by applying the law-of-the-wall

$$
u_{\tau}=\sqrt[3]{\epsilon(y) k y}
$$

(valid close to the wall and/or in high-speed flows) where $\epsilon$ is the turbulent viscous dissipation and $k$ is the Von Kármán constant $(k=0.41)$, to dissipation measurements at $y=10 \mathrm{~cm}$ (Figure 5b) extracted with the inertial dissipation method. Despite the good agreement with the LES on the mean current (Figure 4a), the predicted dissipation levels are systematically higher than the measured ones. However, the dissipation calculated at $y=10 \mathrm{~cm}$ from the LES is consistent, via the law-of-the-wall equation (16), with the total wall-shear stress (Figure 5a), which is calculated independently. A log-law fit of the velocity profiles has also been successfully used to estimate the wall-shear stress, therefore, showing the self-consistency of the simulation. An optimal value of the bottom-drag coefficient $C_{B}$, defined as

$$
u_{\tau}=\sqrt{C_{B}} \sqrt{U_{B}^{2}+W_{B}^{2}} .
$$

was found to be $C_{B}=0.0036$ for the present case. The greatest discrepancies are visible after re-transition when using the law-of-the-wall and the bottom-drag coefficient.

[37] The successful adoption of the aforementioned loworder models for the wall-shear stress is consistent with the absence of nonequilibrium effects in the momentum transport. These typically occur in low-speed (or intermittently turbulent) oscillating currents [Hino et al., 1983; Spalart and Baldwin, 1987; Jensen et al., 1989; Costamagna et al., 2003 ] in the form of violent production of turbulent kinetic 
energy at the end of the deceleration phase, break down of the validity of the log-law during the acceleration phase and visible phase lags between dissipation and the mean component of current associated with the base frequency of the seiche-induced velocity (imbalance between dissipation and production). The numerical results show some of aforementioned events only in very limited portions of the cycle, as discussed later.

[38] Nonequilibrium effects in geophysical flows have been discussed in Lorke et al. [2002] and Lorke et al. [2003], who looked at low-speed oscillating currents in Lake Alpnach. Relatively low current intensities determine longer propagation times throughout the water column of turbulence periodically generated in the near-wall region. This results, for example, in the current intensity leading turbulent dissipation, as observed by Lorke et al. [2003] at $y=1$ $\mathrm{m}$, with a phase lag of $1-2 \mathrm{~h}$. This is a low-Reynolds number effect and is not present in the flow analyzed here. Also, they observe, at $y=1 \mathrm{~m}$ from the bottom, current amplitudes and dissipation values of the same order as the ones observed by Bryant et al. [2010] at $y=10 \mathrm{~cm}$. This implies that the flow analyzed here should be much more energetic than what is suggested by the dissipation measurements (also implied by the higher dissipation predicted by the LES). Partial turbulent kinetic energy transfer to irreversible diapycnal mixing (that could justify this apparent underestimation of the viscous dissipation) has not likely occurred, given that the temperature measurements in Bryant et al. [2010] show no significant stratification at $y=10 \mathrm{~cm}$.

[39] While the magnitude of the measured dissipation may raise some questions [Lorke and Wüest, 2004], its trend and overall (4-orders-of-magnitude) variation is consistent with the variations in the independently measured DO field and SOU (shown later). The numerical model does not predict a reduction in the overall turbulent activity of the same intensity as shown by the measurements. This is a first-order hydrodynamic effect, which is expected to be reproducible even in the idealized conditions adopted in the present LES. This is the main reason for the discrepancies between the measured and simulated SOU and DO field predictions, which are mostly concentrated near flow reversal. In the following, the interaction between the intensity of turbulent mixing and the DO concentration dynamics are discussed and this issue is further investigated.

\subsubsection{Water Column Turbulent Transport}

[40] Figure 6 shows the wide range of scales associated with the transport events in the near-wall region. The energy required for sustaining turbulent mixing and transport across the water column is provided by the mean current, which is initially directed eastward. The current decelerates almost completely between $t=4 \mathrm{~h}$ and $t=6 \mathrm{~h}$, and then reaccelerates changing direction (heading south). Turbulence production will therefore inject energy from the large scales (mostly) into the $u^{\prime}$ and $w^{\prime}$ fluctuations, respectively, before and after flow reversal, as shown by the distributions of the turbulent kinetic energy production and Reynolds shear stresses (Figures 6a-6c) and the resulting turbulent intensity levels (Figures 6d-6f).

[41] The gradual compression of the viscous sublayer due to the initial acceleration (towards east) for $0<t<2 \mathrm{~h}$ is visible in the $\left\langle u^{\prime} v^{\prime}\right\rangle$ Reynolds shear stress distribution and in the RMS of velocity fluctuations, both reaching the maximum

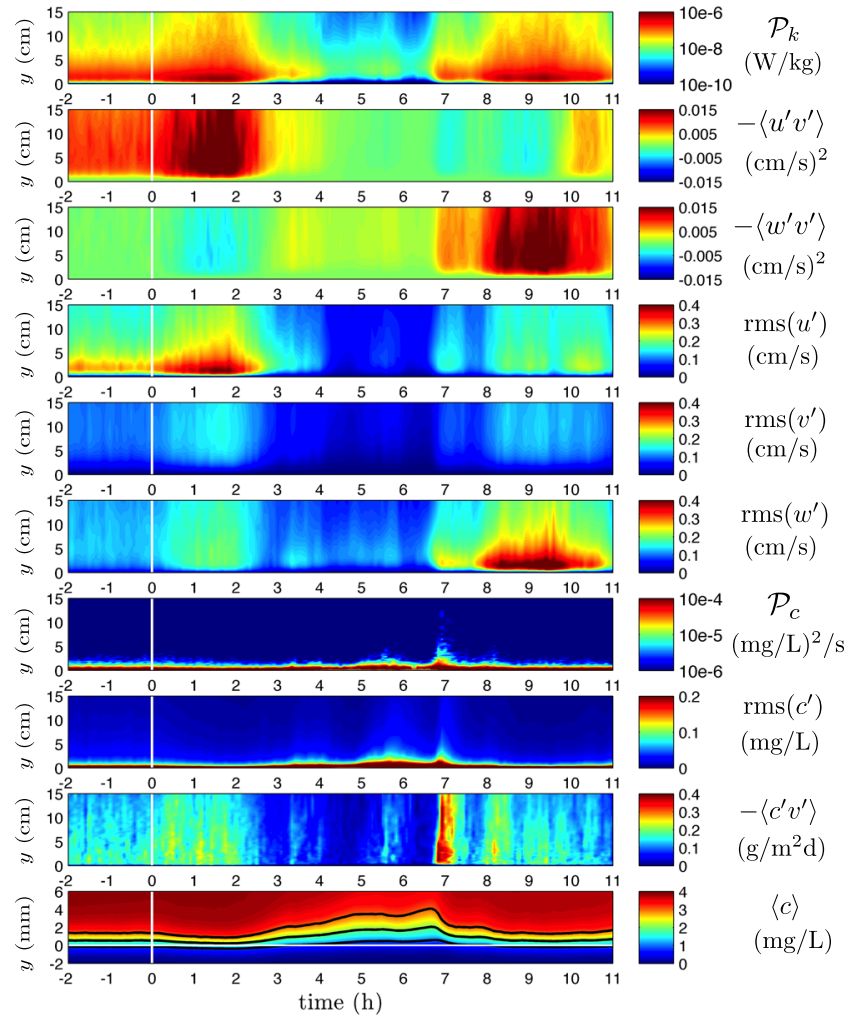

Figure 6. Contour plots showing time history (from top to bottom) of production of turbulent kinetic energy, $\mathcal{P}_{k}=-2\left\langle u_{i}^{\prime} u_{j}^{\prime}\right\rangle \partial\left\langle u_{i}\right\rangle / \partial x_{j}$, Reynolds shear stresses $-\left\langle u^{\prime} v^{\prime}\right\rangle$ and $-\left\langle w^{\prime} v^{\prime}\right\rangle$, RMS of $u^{\prime}, v^{\prime}, w^{\prime}$, production of scalar variance $\mathcal{P}_{c}=-2\left\langle c^{\prime} u_{j}^{\prime}\right\rangle \partial\langle c\rangle / \partial x_{j}$, RMS of $c^{\prime}$, turbulent mass flux $-\left\langle c^{\prime} v^{\prime}\right\rangle$, and mean oxygen concentration $\langle c\rangle$. Isolines of oxygen concentration in the bottom panel are for 1,2 , and $3 \mathrm{mg} \mathrm{L}^{-1}$. Statistical steady state flow used for initial condition $(t<0)$ and seiche-driven unsteady flow $(t>0)$.

intensity closest to the SWI between $t=1.5 \mathrm{~h}$ and $t=2 \mathrm{~h}$. At this time, the signature of the (milder) acceleration towards north (Figure $4 \mathrm{a}$ ) is also visible in the $\left\langle w^{\prime} v^{\prime}\right\rangle$ Reynolds stress distribution and RMS of $w^{\prime}$. The overall enhanced Reynolds shear stresses increase the rate of exchange of momentum with the no-slip boundary and the turbulent mixing. The turbulent mass transfer rate in the water column, $\left\langle c^{\prime} v^{\prime}\right\rangle$ (Figure 6i) increases accordingly due to the enhanced $v^{\prime}$ (Figure 6e) rather than the $c^{\prime}$ (Figures 6g and 6h). This causes fluid rich in oxygen to be pumped towards the SWI resulting in the compression of the diffusive sublayer, visible in the oxygen concentration contours (Figure 6j) and RMS of concentration fluctuations $c^{\prime}$ (Figures 6h), both following closely the initial gentle flow acceleration.

[42] At this stage, there is no visible phase lag between the seiche-induced forcing, the enhanced turbulent activity across the water depth, and the evolution of the diffusive sublayer. The turbulent and molecular momentum and mass fluxes are, with good approximation, in equilibrium with the main current. During the following deceleration stage $(2 \mathrm{~h}<t<6 \mathrm{~h})$, the effects of turbulent transport have 
almost completely vanished by $t=3 \mathrm{~h}$, remaining negligible until $t=6 \mathrm{~h}$. During the whole period of reduced turbulent activity, the diffusive sublayer exhibits a slow but steady expansion, starting between $t=2 \mathrm{~h}$ and $t=3 \mathrm{~h}$, limited by the long diffusive time scales of DO. This is consistent with the conceptual model for the near-wall transport described in Scalo et al. [2012b] where the same behavior is observed in the small-scale turbulent transport events in a fully developed turbulent flow.

[43] The re-acceleration phase for $t>6 \mathrm{~h}$ is less regular. Most of the energy for mixing is provided by the main current directed south (Figures 6b, 6c, and 6f); accordingly, the near-wall turbulent structures responsible for mixing are re-oriented along $z$, feeding energy into the RMS of $w^{\prime}$. The flow does not exhibit the same degree of equilibrium observed before, which results in pronounced phase lags among the different transport processes involved.

[44] Two abrupt turbulent production events, leading to a rapid enhancement of the mixing in the water column, are predicted around $t=6.9 \mathrm{~h}$ and $t=8.1 \mathrm{~h}$ (Figures 6c, 6g, and $6 \mathrm{i}$ ) are not related to the background $8 \mathrm{~h}$ period seiche but to a $2 \mathrm{~h}$ period mode with current amplitude of approximately $0.3 \mathrm{~cm} \mathrm{~s}^{-1}$, visible after flow reversal (Figure $4 \mathrm{a}$ ), from $t=6 \mathrm{~h}$ to $t=9 \mathrm{~h}$. This is consistent with a longitudinal mode 3 surface seiche [Boegman, 2009].

[45] Abrupt turbulent production events are triggered towards the end of the rapid flow acceleration phases, from $t=6 \mathrm{~h}$ to $t=6.9 \mathrm{~h}$ and from $t=7.5 \mathrm{~h}$ to $t=8.1 \mathrm{~h}$, primarily directed south (Figure 4a). The wall-shear stress exhibits no visible delay with respect to the current evolution suggesting that the energy for mixing, in this case, may be provided by the same mechanisms observed in rapidly oscillating, low-speed turbulent boundary layers (in the intermittent/ nonequilibrium regime). During the acceleration phase, the viscous sublayer is compressed (directly enhancing the wall-shear stress) and turbulent production is frozen. Nearwall locations of below- and above-average instantaneous velocity-low-speed and high-speed streaks - then appear. These streaks undergo instability, which results in production and upward propagation of turbulent fluctuations at the beginning of the following deceleration phase [Jimenez and Pinelli, 1999; Costamagna et al., 2003]. This is consistent with the violent ejection of scalar variance from the edge of the diffusive sublayer (Figures $6 \mathrm{~g}$ and $6 \mathrm{~h}$ ) and intensity (Figure 6h) distributions, leading to a sudden increase in the water-column mass flux. During the initial acceleration stage $(0<t<2 \mathrm{~h})$, the enhancement of mixing in the water column is, rather, connected to the increase of RMS of $v^{\prime}$ and occurring under quasi-equilibrium conditions.

[46] The resulting enhanced turbulent mass flux causes a rapid thinning of the diffusive sublayer and, therefore, the enhancement of the DO transfer across the SWI. This occurs with a delay of $\sim 20$ min (shown later), corresponding to a phase lag of $\phi \sim 60^{\circ}$ in a $2 \mathrm{~h}$ period oscillation. The flow reverts, after $t=8.5 \mathrm{~h}$, to a state very similar to the initial conditions, with fully sustained, quasi-steady, turbulent mixing and mass transfer across the SWI.

\subsubsection{A Low-Order Model for the SOU}

[47] The signature of the transitional hydrodynamics is visible in the instantaneous DO distribution across the SWI or, equivalently, in the corresponding SOU time series, both analyzed in the following.
[48] The effects of the flow acceleration (from $t=0$ to $t=2 \mathrm{~h}$ ) are visible in the thinning of the diffusive sublayer. In this phase, the LES follows closely the observations (Figure 7a). During the initial stage of the deceleration phase $(2 \mathrm{~h}<t<4 \mathrm{~h})$ the diffusive sublayer thickness predicted by the numerical model increases faster than the observations (Figures 7b-7d), following closely the reduced intensity of the overlying turbulent transport (Figure 6). Agreement is recovered at $t=4.8 \mathrm{~h}$ where the observed and predicted DO flux across the SWI match. Here the DO profiles begin to lose a well-defined turbulent structure, tending towards a laminar-like (linear) distribution in the water column. While the observed diffusive sublayer starts to expand later than in the LES predictions, it does so more rapidly until $t=6 \mathrm{~h}$, right before flow reversal (Figures $7 \mathrm{~d}$ and 7e). A higher value of the DO flux across the SWI is predicted by the LES. The low molecular diffusivity of DO becomes an important limiting factor for the rate of expansion of the diffusive sublayer in the absence of turbulent forcing. Complete retransition is evident from the DO profiles for $t>6 \mathrm{~h}$ when the current is re-accelerating towards south (Figures $4 \mathrm{~b}$ and $6 \mathrm{c}$ ), and good agreement with the field measurements is recovered.

[49] The proposed SOU model (equation (11)) conceptually separates purely hydrodynamic effects $(\beta)$ from biogeochemically limited quantities $(\Delta C)$. While the former can be successfully modeled with equilibrium assumptions, it is still not clear whether the same approach for $\Delta C$ can lead to acceptable errors in field-scale applications. The available numerical and field data are used in the following to address this issue and, in particular, to determine the functional relationship (equation (14)).

[50] Figure 8 shows LES data from the present transient simulation, the statistically steady lab-scale open-channel simulations by Scalo et al. [2012b], and DO data extracted from field measurements in central Lake Erie (Bouffard, personal communication, 2013). The latter has been obtained by solving the unsteady 1D RANSE equations in the water column coupled with the DO absorption model by Higashino et al. [2008] with free-stream conditions matching the observations. Overall, the data cover a wide range (over 4 orders of magnitude) of $\mu^{+}$. The numerical bounds for $f$ in equation (14) are $f\left(\mu^{+} \rightarrow 0\right)=0$ and $f\left(\mu^{+} \rightarrow+\infty\right)=1$ for every $S c, \varphi$, and $K$. Moreover, the trend of the data in the semi-log plot suggests an asymptotic behavior for $f$ in the two extreme cases. This leads to the approximate functional form

$$
\Delta C / C_{\infty}=\frac{1}{2}\left[\tanh \left(\alpha_{1}\left[\log _{10}\left(\mu^{+}\right)+\alpha_{2}\right]\right)+1\right]
$$

where $\alpha_{1}$ and $\alpha_{2}$ are dimensionless fitting coefficients dependent from $S c, \varphi$, and $K$. This heuristic assumption is also supported by the observed asymptotic tendency of $C_{s w i}$ toward $C_{\infty}$ for increasing wall-shear stress in laboratory and numerical experiments [Higashino et al., 2008; Scalo et al., 2012b], for fixed sediment layer parameters. In the case of more complex sediment biochemistry, the maximum oxidation rate in equation (15) could be replaced with the overall effective DO absorption rate.

[51] The present data set is for one value of porosity $(\varphi=0.95)$, permeability $\left(K=2 \times 10^{-7} \mathrm{~cm}^{2}\right)$, Schmidt number $(\mathrm{Sc} \sim 1000)$, and oxidation rate $\left(\mu=240 \mathrm{mg} \mathrm{L}^{-1} \mathrm{~d}^{-1}\right)$, 

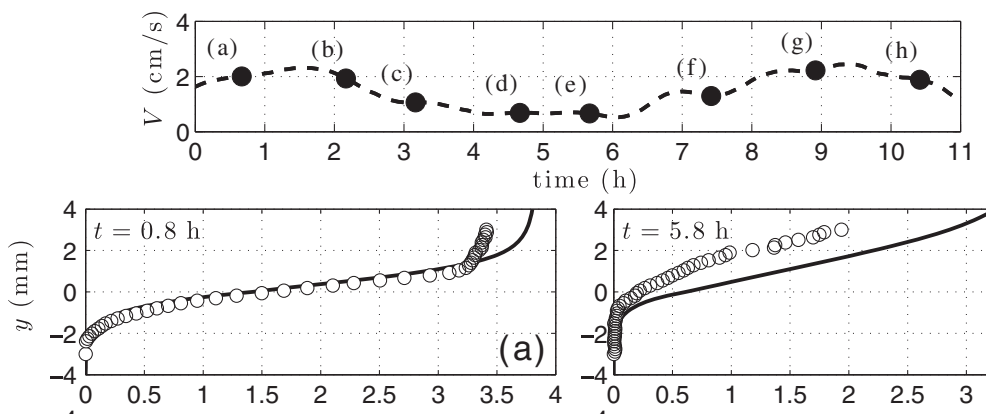

(a)
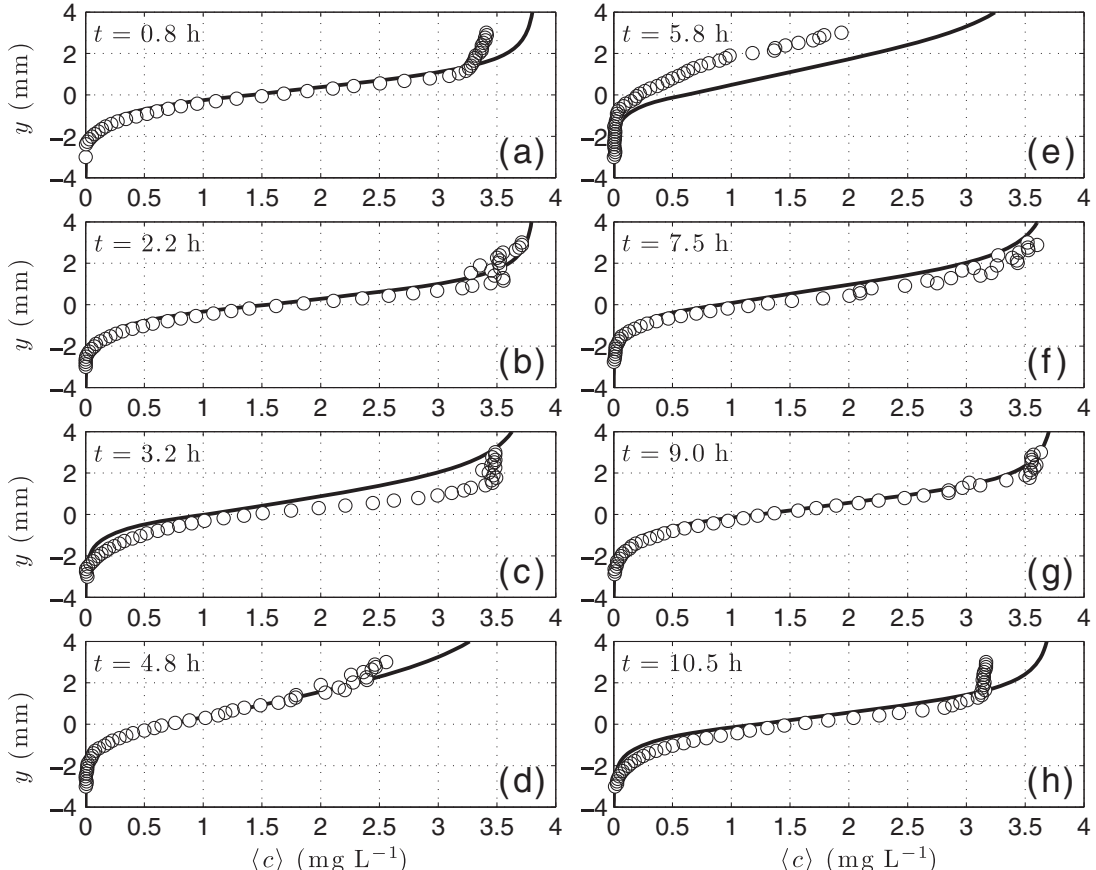

Figure 7. Plane-averaged oxygen concentration profiles extracted at four different times (a-d) before flow reversal $(t<6 \mathrm{~h})$ and $(\mathrm{e}-\mathrm{h})$ after flow reversal $(t>6 \mathrm{~h})$; oxygen measurements obtained via microprofiling by Bryant et al. [2010] (०). Top panel: magnitude of mean velocity vector, $V$, at $10 \mathrm{~cm}$ from the SWI.

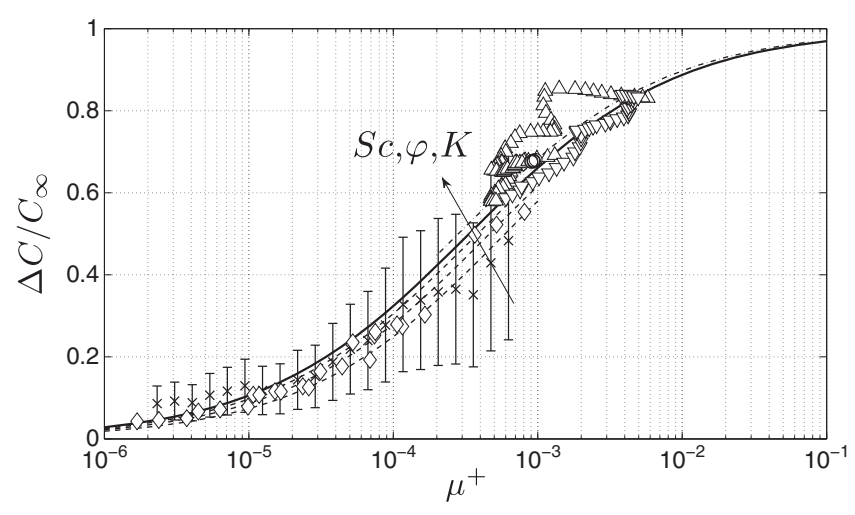

Figure 8. Normalized oxygen difference $\Delta C$ at the SWI as a function of $\mu^{+}$(15): data from Scalo et al. [2012b] for $S c=$ 400,690 , and 1000 , different current intensities $U_{B}=2.5-$ $27 \mathrm{~cm} \mathrm{~s}^{-1}$ and bacterial populations, $\chi=100-700 \mathrm{mg} \mathrm{L}^{-1}$ $(\diamond)$ and corresponding fit for each $S c(---)$, data from the present LES in the acceleration stages $0<t<2 \mathrm{~h}$ and $6 \mathrm{~h}<t<11 \mathrm{~h}(\triangle)$, deceleration stages $2 \mathrm{~h}<t<6 \mathrm{~h}(\nabla)$ and equilibrium conditions, $t<0 \mathrm{~h} \mathrm{(o)}$, and corresponding fit (18) for $\alpha_{1}=0.7$ and $\alpha_{2}=3.55$ (-. - ), data from Lake Erie (Bouffard, personal communication, 2013) $(\times)$ with $95 \%$ confidence interval shown with vertical error bars, proposed overall fit (18) for $\alpha_{1}=0.7$ and $\alpha_{2}=3.475$ (-). The arrow shows the data trend for increasing Schmidt number, porosity, and permeability. in nonequilibrium $(t>0)$ and equilibrium $(t<0)$ conditions for the mass transfer. The data from Scalo et al. [2012b] cover three different Schmidt numbers $(S c=400$, 690 , and 1000 , respectively, for water temperatures of $25^{\circ} \mathrm{C}$, $15^{\circ} \mathrm{C}$, and $\left.8^{\circ} \mathrm{C}\right)$, two different oxidation rates $(\mu=240$ and $\left.1680 \mathrm{mg} \mathrm{L}^{-1} \mathrm{~d}^{-1}\right)$, a wide range of current intensities $\left(U_{B}=\right.$ $\left.2.5-27 \mathrm{~cm} \mathrm{~s}^{-1}\right)$, all for a lower porosity $(\varphi=0.55)$, zero permeability $(K=0)$ and in statistically steady conditions. This explains why the latter data set exhibits a clearer trend, in which Schmidt number effects may also be quantified; a least square fit of equation (18) returns $\alpha_{1}=0.71-0.70$ and $\alpha_{2}=3.2-3.4$ for $S c=400-1000$.

[52] While the LES data shown here include low and high porosity cases, the limited range of permeabilities investigated (between zero and $K=2 \times 10^{-7} \mathrm{~cm}^{2}$ ) does not allow for a robust parametrization of the effects of hyporheic exchange in the proposed low-order model. However, it is not clear if higher permeabilities are realistic for a sediment layer saturated with organic matter [Scalo et al., 2012a]. Low bacterial populations $\chi$ are expected to be correlated with higher permeabilities as in the present case. The molecular component of the DO flux modeled by equation (11) is dominant in deep waters. However, a purely advective component of the flux across the SWI, for example, induced by wave transport near the shoreline, can be linearly superimposed (Brennen and Imberger, personal communication). A more robust hyporheic exchange parametrization, such as the one proposed by Grant et al. [2012] 
(sharing a similar functional form as equation (7)) could be incorporated in the present model, although strong assumptions regarding the resulting formulation of the parameterized flux, especially at the interface, would have to be made. For a detailed discussion on this issue, see Thibodeaux [2011] and Thibodeaux et al. [2012].

[53] Analogous data from the present simulation are characterized by a pronounced hysteresis caused by the transitional flow. However, values of $\Delta C$ for $t<0$ can still be used to infer the fitting coefficients for the corresponding equilibrium conditions, resulting in $\alpha_{1}=0.7$ and $\alpha_{2}=3.55$. This parametrization splits the data in two parts: one relative to the acceleration stages, for $0<t<2 \mathrm{~h}$ and $6 \mathrm{~h}$ $<t<11 \mathrm{~h}$, where the inertia of the diffusive sublayer retains higher $\Delta C$ than equilibrium conditions, and another one to the deceleration stages, for $2 \mathrm{~h}<t<6 \mathrm{~h}$, where $\Delta C$ is lower than equilibrium conditions. The fit leads to higher equilibrium values for $\Delta C$ than the other data. This is due to the relatively higher porosity and permeability of the present case, as shown in Scalo et al. [2012a] and Higashino and Stefan [2011] and previously discussed in Figure 2.

[54] For both data sets, the effects of $\mu^{+}$are dominant. The Schmidt number dependency in equation (14) highlights its effects on the diffusive sublayer thickness (limiting the mass transfer rate from the water side). As anticipated in section 2.3, there are not many elements at this stage of the model development to account explicitly for the dependency on porosity and permeability. Their effects on $\Delta C$ can, however, still be predicted and understood. This motivates us to further simplify the model considering only lower temperatures $(\mathrm{Sc} \sim 1000)$, which are more representative of conditions found in bottom layers of oceans and lakes, only equilibrium conditions, and averaging between the low and high porosity data. The final parameterization proposed for $\Delta C$ results in values of $\alpha_{1}=0.7$ and $\alpha_{2}=3.475$. Although we believe that the assumptions made so far lead to errors within acceptable bounds, they are, for now, only heuristic and require further empirical confirmation.

[55] Finally, the predictions of the proposed model are compared against the sediment-oxygen uptake directly calculated from the LES (Figure 9). The mass-transfer coefficient (equation (13)) was calculated using the parametrization by Shaw and Hanratty [1977] $(A=0.0889$ and $n=0.704$, the closest to the SOU predictions in Scalo et al. [2012b]), together with the bottom-drag-coefficient approximation of the total friction velocity (equation (17)). Despite the simplicity of the adopted model, the overall SOU variability predicted by the LES is correctly captured. The observable discrepancies are primarily due to the equilibrium assumptions made in the formulation for $\beta$ and $\Delta C$.

[56] The main discrepancy with the measurements in Bryant et al. [2010] lies in the amplitude of the excursion of the SOU. As discussed in section 3.2.1, this is a natural effect of the differences between the dissipation predicted by LES and the corresponding measurements (Figure 5b). The latter, however, are not entirely consistent with the measured current velocity and the assumptions regarding the adoption of the log-law for the velocity profiles.

[57] The LES predicts time lags between the SOU and the turbulent forcing (or $u_{\tau}$ ). These are due to the long time scales of the growth and compression of the diffusive

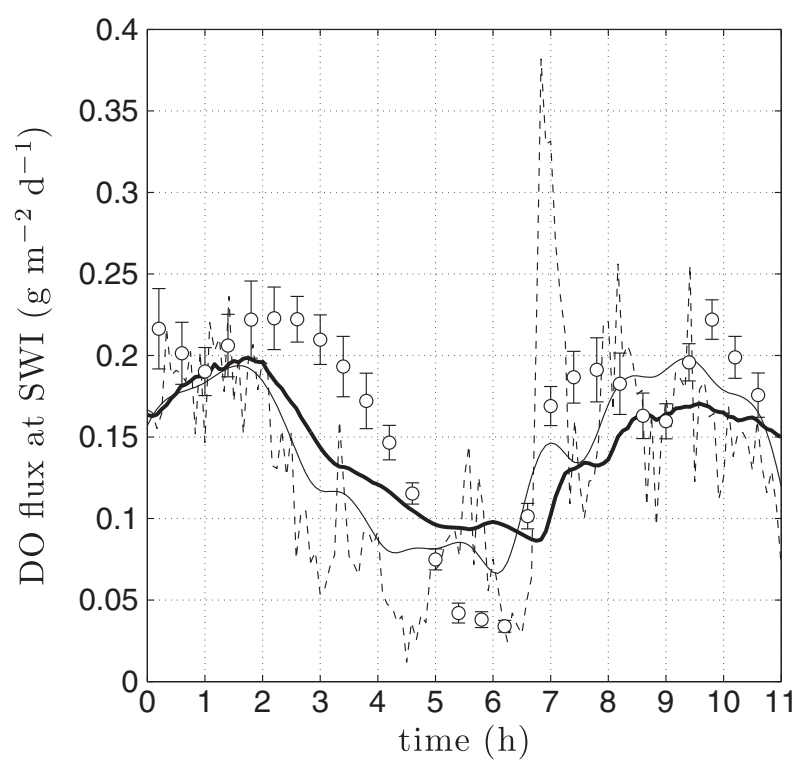

Figure 9. Time series of vertical DO flux. SOU directly computed from LES ( $\longrightarrow$ ), proposed parametrization (11) based on equations (17), (18), and (13) for $A=0.0889$ and $n=0.704$ [Shaw and Hanratty, 1977] (-), measurements by Bryant et al. [2010] (o), turbulent DO flux $-<c^{\prime} v^{\prime}>$ at $y=1 \mathrm{~cm}$ from the SWI (-- ).

sublayer and cannot be accounted for in the proposed algebraic SOU model. For example, Figure 9 shows how during the deceleration phase, from $t=2 \mathrm{~h}$ to $t=6 \mathrm{~h}$, the SOU model predicts a more rapid expansion of the diffusive sublayer (more rapid drop in the DO flux) than the LES. This is a direct consequence of the equilibrium assumptions made in the algebraic model, where the state of turbulent mixing is parameterized based on the wall-shear stress (see equations (13) and (15)). The latter does not exhibit a significant delay with respect to the main forcing and neither will the modeled SOU. The LES, on the other hand, resolves the time evolution of the diffusive sublayer, capturing its inertia with respect to the turbulent forcing (see also discussion in Scalo et al. [2012a]), thus retaining a higher value of the flux for a longer time despite the sudden drop in $u_{\tau}$. Analogously, the delay of $\sim 20-30 \mathrm{~min}$, in the SOU enhancement predicted by the LES, following the mixing events in the water-column at $t=6.9 \mathrm{~h}$ and $t=8.1 \mathrm{~h}$, is not captured by the SOU model.

[58] Equilibrium conditions for the scalar transport are observed for the majority of the seiche cycle, in our case, away from the SWI where the predicted turbulent mass flux follows closely the Reynolds stresses and turbulent kinetic energy distribution but with a remarkably higher variance (Figure 6i). This is consistent with the intrinsically intermittent nature of a passively advected scalar in a turbulent flow [Warhaft, 2000], which is enhanced in the case of a highSchmidt-number contaminant [Antonia and Orlandi, 2003]. The same behavior has been observed by Brand et al. [2008] in eddy-correlation measurements for a similar oscillating flow in Lake Alpnach. The DO flux on the water side is characterized by a broader range of temporal and spatial scales and overall variance that can exceed the SOU itself (Figure 9). 
[59] Although Bryant et al. [2010] have not observed any delay between the near-wall turbulent activity and the SOU response, Lorke et al. [2003] have shown a well defined time lag between the Batchelor scale (extrapolated at the SWI from dissipation measurements taken at $y=0.5 \mathrm{~m}$ ) and the estimated diffusive sublayer thickness. They observed an abrupt reduction of the near-wall turbulent activity followed by a gradual relaxation of the diffusive sublayer. Abrupt increases in turbulent activity can precede by more than an hour the corresponding enhancement of the SOU, depending on the time scale and intensity of the driving current. The same behavior has been observed in the present numerical experiments (Figures 6 and 9).

\section{Conclusions}

[60] We have adopted a previously developed model based on large-eddy simulation (LES) to investigate the governing transport mechanisms involved in DO transfer from a turbulent flow to an organic sediment bed. An idealized numerical experiment has been carried out with biogeochemical and flow conditions inspired by the measurements in Lake Alpnach of Bryant et al. [2010] capturing a full seiche cycle. The prediction of turbulence decay and transition for the velocity field in LES is rather sensitive to initial conditions, which cannot be determined to the level of accuracy required by the model for the particular flow under investigation. The LES could not be designed with the intention to match exactly the field measurements and the observed discrepancies were expected.

[61] The LES does not predict the pronounced decay of turbulence during flow reversal observed in the field-scale dissipation measurements. However, the statistics of the synthetically reproduced near-wall turbulence and instantaneous DO field are quantitatively consistent with the seiching current (primary input driving the hydrodynamics of the model), which is not the case for the field measurements. The high degree of uncertainty of the measured data in the field has resulted in the latter being used, rather, as a reference to derive via LES a more controlled and self-consistent data set for a geophysical transitional flow of this kind.

[62] In spite of the simplified computational setup, a wide spectrum of coupled physical processes present in a realistic geophysical environment have been reproduced. These include seiche-driven mixing, turbulent decay, retransition, diffusion of DO across the SWI, and transport and absorption in the pore water.

[63] The understanding of these mechanisms acquired so far only through laboratory measurements has been employed to assist field-scale modeling. The challenges that (statistically) unsteady turbulence poses to modeling have been discussed. For this particular flow, nonequilibrium effects were not observed in the momentum transport but rather in the high-Schmidt-number mass transfer dynamics. It has been shown that this resulted in a straightforward prediction of the wall-shear stress with low-order models such as the law of the wall, log-law fitting of the velocity profile, and the drag coefficient but in a more difficult parametrization of the SOU.

[64] The artificially prescribed conditions made it possible to assess accurately the sensitivity and response of the SOU to the aforementioned processes and provide high-fidelity data for its low-order modeling. The data from this study, together with previously obtained lab-scale LES and results from other field measurements, have been employed to develop a process-oriented algebraic model for the SOU. The latter is parameterized as the product of a mass transport coefficient $\beta$, modeling the effects of turbulent mixing, and the oxygen concentration defect at the SWI $\Delta C$, modeling the biogeochemical limiting component. $\Delta C$ is parameterized assuming equilibrium between near-wall turbulent transport and absorption in the sediment layer. The only input parameters required are the sediment oxidation rate, bulk temperature and DO concentration, and friction velocity.

[65] Despite the assumptions made, the overall agreement of the algebraic model for the SOU with the LES is remarkable. The model should, however, also be tested in flows such as the one investigated by Lorke et al. [2003] where nonequilibrium effects are present at all stages of transport (complete oxygen profiling at all phases was, unfortunately, not provided by the authors). It is expected for flows of this type that the model still predicts the correct value of the mean flux over a seiche cycle.

[66] Limitations of the proposed SOU model include the nonexplicit dependency of the flux from porosity and permeability, which can affect both $\beta$ and $\Delta C$. This can be improved by considering more advanced hyporheic exchange laws. Numerical and experimental investigations, systematically covering a wide range of porosities, permeabilities, and different bed forms and roughnesses would have to be carried out to provide accurate data to validate them. This is computationally very challenging. The proposed model applicability is limited to the case of a twolayer exchange between water and flat cohesive sediments, where the limiting factor for the mass transfer is a diffusive sublayer at the interface. The model is not expected to perform well in cases where the advective component of the flux across the SWI is dominant. However, for the case of oxygen transfer and depletion, it is not clear whether sediment beds containing organic matter can reach sufficiently high permeabilities for pore-water-flow induced transport to be important. A first step would be to quantify the dependency of the sediment layer porosity from the organic content, as it is dominant over permeability effects.

[67] In conclusion, we believe that the conceptual framework supporting the proposed parametrization is applicable in the vast majority of scenarios where oxygen depletion is occurring and can provide a first tentative definition of simple process-oriented models for the SOU, currently lacking in water-quality management applications.

[68] Acknowledgments. We acknowledge the financial support of the Natural Science and Engineering Research Council of Canada under the Discovery Grant Program and the Canada Research Chair program. The authors also thank the High Performance Computing Virtual Laboratory (HPCVL), Queen's University site, for the computational support. The authors thank Damien Bouffard for the fruitful discussions on the flow physics, field-scale measurements, on the parametrization of the sediment-oxygen uptake and for providing us with his field data.

\section{References}

Antonia, R., and P. Orlandi (2003), Effect of Schmidt number on smallscale passive scalar turbulence, Appl. Mech. Rev., 56, 615-632, doi: http://dx.doi.org/10.1115/1.1581885.

Bear, J. (1972), Dynamics of Fluid in Porous Media, American Elsevier, New York. 
Boegman, L. (2009), Currents in stratified water bodies 2: Internal waves, in Encyclopedia of Inland Waters, edited by E. in Chief: Gene E. Likens, Academic Press, Oxford, 539-558, doi:10.1016/B978012370626-3.00081-8, (to appear in print).

Boegman, L., M. Loewen, P. Hamblin, and D. Culver (2008a), Vertical mixing and weak stratification over zebra mussel colonies in western Lake Erie, Limnol. Oceanogr., 53 (3), 1093-1110, doi: 10.4319/1o.2008.53.3.1093

Boegman, L., M. R. Loewen, D. A. Culver, P. F. Hamblin, and M. N. Charlton (2008b), Spatial-dynamic modeling of algal biomass in Lake Erie: Relative impacts of Dreissenid mussels and nutrient loads, J. Environ. Eng-ASCE, 134 (6), 456-468, doi: 10.1061/(ASCE)07339372(2008)134:6(456)

Boudreau, B., and B. Jørgensen (2001), The Benthic Boundary Layer: Transport Processes and Biogeochemistry, Oxford University Press, New York.

Brand, A., D. F. McGinnis, B. Wehrli, and A. Wüest (2008), Intermittent oxygen flux from the interior into the bottom boundary of lakes as observed by eddy correlation, Limnol. Oceanogr., 53(5), 1997-2006, doi: 10.4319/lo.2008.53.5.1997.

Bryant, L. D., C. Lorrai, D. F. McGinnis, A. Brand, A. Wüest, and Little, J. C. (2010), Variable sediment oxygen uptake in response to dynamic forcing, Limnol. Oceanogr., 55 (2), 950-964, doi: 10.4319/lo.2010.55.2.0950.

Burns, N. M., and C. Ross, (1972), Paper no. 6, USEPA Tech. Rept. TS-0571-208-24, Canada Centre for Inland Waters, Burlington, Ontario.

Committee on Environment and Natural Resources, (2010), Scientific assessment of hypoxia in U.S. coastal waters, Tech. Rep., Interagency Working Group on Harmful Algal Blooms, Hypoxia, and Human Health of the Joint Subcommittee on Ocean Science and Technology.

Conroy, J. D., L. Boegman, H. Zhang, W. J. Edwards, and D. A. Culver (2011), "Dead Zone" dynamics in Lake Erie: the importance of weather and sampling intensity for calculated hypolimnetic oxygen depletion rates, Aquat. Sci., 73(2), 289-304, doi:10.1007/s00027-010-0176-1.

Costamagna, P., G. Vittori, and P. Blondeaux (2003), Coherent structures in oscillatory boundary layers, J. Fluid Mech., 474(1), 1-33, doi: $10.1017 /$ S0022112002002665.

Germano, M., U. Piomelli, P. Moin, and W. Cabot (1991), A dynamic subgrid-scale eddy viscosity model, Phys. Fluids A, 3, 1760-1765.

Grant, S. B., and I. Marusic (2011), Crossing turbulent boundaries: Interfacial flux in environmental flows, Environ. Sci. \& Techno., 45(17), 7107-7113, doi:10.1021/es201778s.

Grant, S. B., M. J. Stewardson, and I. Marusic (2012), Effective diffusivity and mass flux across the sediment-water interface in streams, Water Resour. Res., 48, W05548, doi:10.1029/2011WR011148

Higashino, M., and H. Stefan (2011), Dissolved oxygen demand at the sediment-water interface of a stream: Near-bed turbulence and pore water flow effects, J. Environ. Eng., 137 (7), 531-540, doi: 10.1061/(ASCE)EE.1943-7870.0000368.

Higashino, M., B. L. O'Connor, M. Hondzo, and H. G. Stefan (2008), Oxygen transfer from flowing water to microbes in an organic sediment bed, Hydrobiologia, 614, 219-231, doi:10.1007/s10750-008-9508-8.

Higashino, M., J. Clark, and H. Stefan (2009), Pore water flow due to nearbed turbulence and associated solute transfer in a stream or lake sediment bed, Water Resour. Res., 45(12), W12,414, doi:10.1029/2008WR007374.

Hino, M., M. Kashiwayanagi, A. Nakayama, and T. Hara (1983), Experiments on the turbulence statistics and the structure of a reciprocating oscillatory flow, J. Fluid Mech., 131, 363-400

Hipsey, M., and D. Hamilton, (2008), Computational aquatic ecosystem dynamic model: CAEDYM v3 science manual, Tech. Rep., Centre for Water Research Report.

Jensen, B. L., B. M. Sumer, and J. Fredsøe (1989), Turbulent oscillatory Reynolds numbers, J. Fluid Mech., 206, 265-297.

Jimenez, J., and A. Pinelli (1999), The autonomous cycle of near-wall turbulence, J. Fluid Mech., 389, 335-359.

Keating, A., U. Piomelli, K. Bremhorst, and S. Nešić (2004), Large-eddy simulation of heat transfer downstream of a backward-facing step, $J$. Turbul., 5(20), 1-27, doi:10.1088/1468-5248/5/1/020.

Kolmogorov, A. N. (1941), The local structure of turbulence in incompressible viscous fluid for very large reynolds number, Dokl. Akad. Nauk SSSR, 30, 9-13.

Koseff, J., J. Holen, S. Monismith, and J. Cloern (1993), Coupled effects of vertical mixing and benthic grazing on phytoplankton populations in shallow, turbid estuaries, J. Mar. Res., 51(4), 843-868.

Leon, L., R. Smith, J. Romero, and R. Hecky (2006), Lake Erie hypoxia simulations with ELCOM-CAEDYM, in The 3rd Biennial Meeting of the International Environmental Modelling and Software Society, Burlington, Vermont, July 9-13.

Leon, L. F., R. E. H. Smith, S. Y. Malkin, D. Depew, M. R. Hipsey, J. P. Antenucci, S. N. Higgins, R. E. Hecky, and R. Y. Rao (2012), Nested 3D modeling of the spatial dynamics of nutrients and phytoplankton in a Lake Ontario nearshore zone, J. Great Lakes Res., 38, 171-183, doi:10.1016/j.jglr.2012.02.006.

Leonard, A. (1974), Energy cascade in large-eddy simulations of turbulent fluid flows, Adv. Geophys., 18, 237-248.

Li, Y.-H., and S. Gregory (1974), Diffusion of ions in sea water and in deep-sea sediments, Geochim. Cosmochim. Acta, 38(5), 703-714, doi: 10.1016/0016-7037(74)90145-8.

Lilly, D. K. (1992), A proposed modification of the Germano subgrid-scale closure method, Phys. Fluids A, 4, 633-635.

Lorke, A., L. Umlauf, T. Jonas, and A. Wüest (2002), Dynamics of turbulence in low-speed oscillating bottom-boundary layers of stratified basins, Environ. Fluid Mech., 2 (4), 291-313, doi: 10.1023/A:1020450729821.

Lorke, A., B. Müller, M. Maerki, and A. Wüest (2003), Breathing sediments: The control of diffusive transport across the sediment-water interface by periodic boundary-layer turbulence, Limnol. Oceanogr., 48(6), 2077-2085.

Lorke, A., and A. Wüest (2004), Application of coherent ADCP for turbulence measurements in the bottom boundary layer, J. Atmos. Oceanic Technol., 22, 1821-1828., doi:10.1175/JTECH1813.1.

Matisoff, G., and T. M. Neeson (2005), Oxygen concentration and demand in Lake Erie sediments, J. Great Lakes Res., 31, Supplement $2(0)$, 284-295, Lake Erie Trophic Status Collaborative Study, doi: 10.1016/S0380-1330(05)70321-X.

Moser, R. D., J. Kim, and N. N. Mansour (1999), Direct numerical simulation of turbulent channel flow up to $\mathrm{Re}=590$, Phys. Fluids, 11(4), 943-945, doi:10.1063/1.869966.

O'Connor, B. L., and M. Hondzo (2008), Dissolved oxygen transfer to sediments by sweep and eject motions in aquatic environments, Limnol. Oceanogr., 53(2), 566-578.

Patterson, J., B. Allanson, and G. Ivey (1985), A dissolved oxygen budget model for Lake Erie in summer, Freshwater Biol., 15, 683-694.

Pinczewski, W., and S. Sideman (1974), A model for mass (heat) transfer in turbulent tube flow. Moderate and high Schmidt (Prandtl) numbers, Chem. Eng. Sci., 29(9), 1969-1976.

Richardson, L. F. (1922), Weather Prediction by Numerical Process, Cambridge University Press, London.

Riedl, R. J., N. Huang, and R. Machan (1972), The subtidal pump: A mechanism of interstitial water exchange by wave action, Mar. Biol., 13, 210-221.

Scalo, C., U. Piomelli, and L. Boegman (2012a), Large-eddy simulation of oxygen transfer to organic sediment beds, J. Geophys. Res., 117(C6), C06005, doi:10.1029/2011JC007289.

Scalo, C., U. Piomelli, and L. Boegman (2012b), High-Schmidt-number mass transport mechanisms from a turbulent flow to absorbing sediments, Phys. Fluids, 24, 085103, doi:10.1063/1.4739064.

Shaw, D. A., and T. J. Hanratty (1977), Turbulent mass transfer to a wall for large Schmidt numbers, AIChE J., 23(1), 28-37.

Spalart, P. R., and B. S. Baldwin (1987), Direct simulation of a turbulent oscillating boundary layer, in Turbulent Shear Flows, vol. 6, 417-400.

Thibodeaux, L. J. (2011), The Flux Equations for Mass Transport Processes Across Interfaces, Chap. 4, Francis and Taylor Group, UK.

Thibodeaux, L. J., K. Valsaraj, and D. Reible (2012), Letter to the editor regarding "crossing turbulent boundaries: Interfacial flux in environmental flows", Environ. Sci. Technol., 46, 1293-1294, doi: 10.1021/es300040t.

Trolle, D., H. Skovgaard, and E. Jeppesen (2008), The water framework directive: Setting the phosphorus loading target for a deep lake in Denmark using the 1D lake ecosystem model DYRESM-CAEDYM, Ecol. Model., 219(1-2), 138-152, doi:10.1016/j.ecolmodel.2008.08.005.

van Rijn, L. C. (1984), Sediment transport, part III: Bed forms and alluvial roughness, J. Hydraul. Eng., 110, 1733-1754.

Warhaft, Z. (2000), Passive scalars in turbulent flows, Annu. Rev. Fluid Mech., 32(1), 203-240.

Wuest, A., and A. Lorke (2003), Small-scale hydrodynamics in lakes, Annu. Rev. Fluid Mech., 35(1), 373-412, doi:10.1146/annurev.fluid.35. 101101.161220.

Zhang, H., D. A. Culver, and L. Boegman (2008), A two-dimensional ecological model of Lake Erie: Application to estimate Dreissenid impacts on large lake Plankton populations, Ecol. Model, 214(2-4), 219-241, doi: 10.1016/j.ecolmodel.2008.02.005. 